\section{Children in cars: how are they being restrained?}

The Motor Vehicles (Wearing of Seat Belts) Regulations state that anyone travelling in the front seat of a car must wear a seat belt and any child under the age of 1 in the front seat must be in an approved restraint designed for his age and weight. This legislation does not ensure the safety of children in cars, as any child may travel in the back seat without any restraint, a child over the age of 1 may travel in the front seat in an inappropriate restraint, and there is no control over the anchorage of back seat restraints.

We studied how children are usually restrained in cars. The British Standards Institution ${ }^{1}$ and the Department of Transport ${ }^{2}$ recommend the following restraints for children: for those aged under 9 months $(<10 \mathrm{~kg})$ a carrycot either with a harness or wedged between front and back seats; for those aged 9 months to 4 years $(10-20 \mathrm{~kg})$ a child seat; for those aged $5-9$ years $(20-40 \mathrm{~kg})$ a child seat or adult belt with booster cushion; and for those aged over $9(>40 \mathrm{~kg})$ an adult seat belt. Alternatively, a specifically designed adjustable belt can be used for ages 5-13 years.

We also considered the anchorage of back seat restraints. Most people do not realise that if the rear anchorage point is too near the back of the seat in an estate or hatchback car the wearer can move within the restraint (figure).
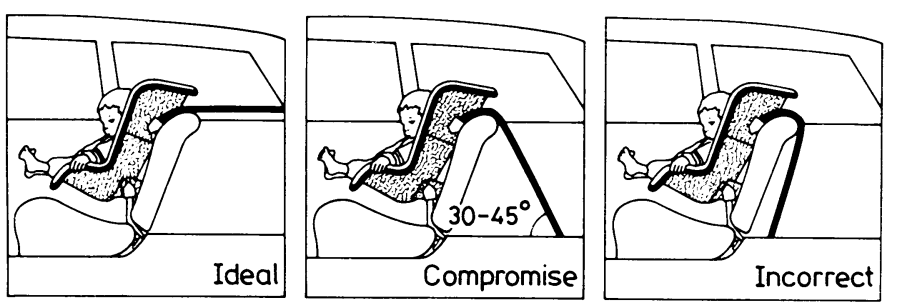

Positioning of rear anchorage points.

\section{Method and results}

We interviewed 130 parents using a standard questionnaire to determine the number and ages of children carried in each car; the type, make, and anchorage of restraints used; and whether the restraints were fitted by the owner of the car or a "professional."

Eight drivers could not give sufficient data for analysis. The remaining 122 carried a total of 268 children. Only 91 children $(34 \%)$ were correctly restrained, $117(44 \%)$ were not restrained, $28(10 \%)$ were in inappropriate restraints, and $34(13 \%)$ were in incorrectly anchored restraints. Full information on anchorage was obtained for 95 of the 108 back seat restraints fitted, of which 34 were incorrectly anchored. There was no difference between those fitted by owners ( 29 correct out of $55(53 \%)$ ) and those fitted by professionals (nine correct out of $24(38 \%)$ ).

Only two children were carried in the front seat in contravention of the current law. One was not restrained at all, and the other was held on an adult's lap with a seat belt around both.

\section{Comment}

In 1983, 577 car and van occupants under the age of 14 were killed or seriously injured. ${ }^{3}$ This figure is $50 \%$ less than that of 1982 , when the seat belt regulations were not in force. The drop is due mainly to a decrease in injury to front seat passengers ( $48 \%$ down), with only a minimal decrease in injuries to rear seat occupants ( $3 \%$ down).

We found that only one third of children under the age of 14 were correctly restrained, yet only two of our cases contravened the present law. Several reasons were given by parents for not using restraints: firstly, the cost; secondly, restraints decrease the amount of space in a car; and, finally, some children dislike being carried in restraints and climb out or suffer from travel sickness when restrained.

What can be done to promote the use of child restraints ? Several studies have emphasised the role of the doctor in educating parents, ${ }^{45}$ but, though counselling helps, it does not appreciably increase the use of child restraints. More effort is needed from those concerned with community health, the media, and those responsible for opinion making and, ultimately, legislation.

1 British Standards Institution. Specification for seat belt assemblies for motor vehicles. Section 5: restraining devices for children. Milton Keynes: British Standards Institution, 1960:14-20. (BSI 3254.)

2 Department of Transport. Child safety in cars 1982. London: HMSO, 1983.
3 Department of Transport, Scottish Development Department, Welsh Office. Road accidents Great Britain 1983. London: HMSO, 1984 .
Pless IB. Accident prevention and health education: back to the drawing board ? Pediatrics $1978 ; 62: 431-5$. 5 Reisinger KS, Williams, AF, Wells JK, John CE, Roberts TR, Podgainy HJ:
Effect of pediatricians' counselling on infant restraint use. Pediatrics $1981 ; 67$ :
201-6. 201-6.

(Accepted 10 fuly 1985)

Department of Child Health, Charing Cross and Westminster Medical School, Charing Cross Hospital, London W6 8RF RUTH EATOUGH, medical student THOREYA SWAGE, BA, medical student PETER WILLSON, BSC, medical student JOHN BROOMHALL, MB, MRCP, lecturer in child health Correspondence to: Dr Broomhall.

\section{Control of an outbreak of systemic Candida albicans}

Recently an outbreak of systemic candidiasis on the intensive care unit at the London Hospital was reported. ${ }^{1}$ From July 1983 to March 1984 a single strain of Candida albicans, defined as serotype A, morphotype $A 1$, biotype $0 / 155 / 7$, caused 12 cases of proved systemic candidiasis, and one suspected case, as defined previously. ${ }^{1}$ The staff transmitted the strain between patients on their hands, with their mouths and perineums acting as secondary reservoirs of infection. Four of the 65 staff were oral carriers, and one nurse carried the strain on her hands. ${ }^{1}$ We report here the outcome of using oral ketoconazole in an attempt to control this outbreak; ketoconazole has been reported as superior to amphotericin B and nystatin ${ }^{2}$ and has been used successfully to treat chronic mucocutaneous candidiasis ${ }^{3}$ and systemic candidiasis. ${ }^{4}$

\section{Patients, methods, and results}

All patients admitted to the intensive care unit from May to October 1984 received oral ketoconazole $400 \mathrm{mg}$ daily until they were discharged. Patients were swabbed orally and perineally on admission and discharge and weekly for carriage of yeast. Oral and perineal carriage in the staff was assessed three months after the treatment with ketoconazole was begun and also at six months, just before it was stopped. All isolates were typed as described previously and confirmed by sonityping. ${ }^{1}$

The outbreak strain proved fairly resistant to Hibiscrub, the disinfectant used at the time of the outbreak. ${ }^{1}$ Thus either Betadine (Napp, United Kingdom) or Hibisol (Imperial Chemical Industries, United Kingdom) was used instead of Hibiscrub when patients with heavy candidal infection were being nursed. The strain proved fully sensitive to both these reagents in handwashing experiments (W Lee, J P Burnie, R C Matthews, unpublished observations).

In April 1984, when an increasing proportion of patients were receiving ketoconazole, there was one proved and three suspected cases of systemic candidiasis, all due to the strain that caused the outbreak. In the next six months, when all patients were receiving ketoconazole, one proved and three suspected cases occurred, and only one of these was caused by the outbreak strain. After treatment was stopped there was one other case, which was caused by a different strain (figure).

The proportion of patients in the unit for over three days who were colonised with the outbreak strain also fell sharply, from $39 \%$ (16 out of 41 patients) before treatment with ketcconazole to $15 \%$ (seven of 47 patients) during treatment. The incidence of colonisation remained low at $17 \%$ (four of 24) after ketoconazole was withdrawn. At this time only two of the oral swabs taken from the 65 staff grew yeasts, and neither was the outbreak strain.

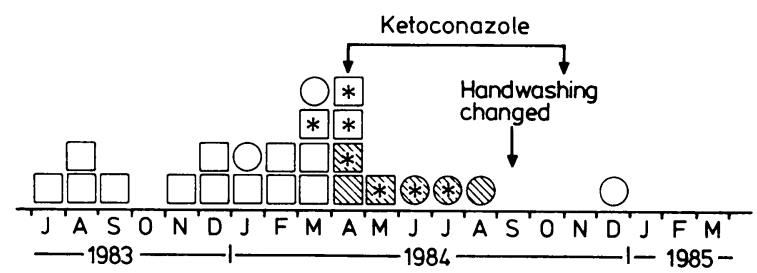

Distribution of cases of systemic candidiasis due to outbreak strain $(\square)$ and non-outbreak isolate (O) over time. Shading indicates prophylaxis with ketoconazole.

* Suspected cases. 